\title{
Pediatric esophageal stenoses: Challenges and new surgical device promoting tension-free esophageal anastomosis
}

\author{
DAN ALEXANDRU IOZSA ${ }^{1,2^{*}}$, RADU-IULIAN SPĂTARU ${ }^{1,2^{*}}$, LUMINITA FLORENTINA TOMESCU $^{3}$, \\ OVIDIU STIRU ${ }^{4,5}$, FLORENTINA GHERGHICEANU ${ }^{6}$, FLORENTINA FURTUNESCU ${ }^{7}$, \\ DANIEL RADAVOI $^{8,9}$, NICOLAE BACALBASA $^{10,11^{*}}$, IRINA BALESCU $^{12}$ and ADRIAN TULIN ${ }^{13,14}$ \\ ${ }^{1}$ Department of Pediatric Surgery, Faculty of Medicine, 'Carol Davila' University of Medicine and Pharmacy, \\ 020021 Bucharest; ${ }^{2}$ Department of Pediatric Surgery, 'Marie S. Curie' Emergency Clinic Hospital for Children, \\ 041451 Bucharest; ${ }^{3}$ Department of Interventional Radiology, 'Prof. Dr. Agrippa Ionescu' Clinical Emergency Hospital, \\ 011356 Bucharest; ${ }^{4}$ Department of Cardiovascular Surgery, 'Prof. Dr. C.C. Iliescu' Emergency Institute for \\ Cardiovascular Diseases, 022322 Bucharest; Departments of ${ }^{5}$ Cardiovascular Surgery, \\ ${ }^{6}$ Marketing and Medical Technology, and ${ }^{7}$ Public Health and Management, \\ 'Carol Davila' University of Medicine and Pharmacy, 020021 Bucharest; ${ }^{8}$ Department of Urology, \\ 'Prof. Dr. Th. Burghele' Clinical Hospital, 050659 Bucharest; ${ }^{9}$ Department of Urology, \\ 'Carol Davila' University of Medicine and Pharmacy, 020021 Bucharest; ${ }^{10}$ Department of Visceral Surgery, \\ 'Fundeni' Center of Excellence in Translational Medicine Clinical Institute, 022328 Bucharest; \\ ${ }^{11}$ Department of Obstetrics and Gynecology, 'Carol Davila' University of Medicine and Pharmacy, 020021 Bucharest; \\ ${ }^{12}$ Department of Surgery, 'Ponderas' Academic Hospital, 021188 Bucharest; ${ }^{13}$ Department of Anatomy, \\ Faculty of Medicine, 'Carol Davila' University of Medicine and Pharmacy, 020021 Bucharest; \\ ${ }^{14}$ Department of General Surgery, 'Prof. Dr. Agrippa Ionescu' Clinical Emergency Hospital, 011356 Bucharest, Romania
}

Received October 26, 2021; Accepted November 25, 2021

DOI: $10.3892 / \mathrm{etm} .2022 .11144$

\begin{abstract}
Esophageal stenoses of childhood have a broad spectrum of underlying causes. Their treatment is usually minimally invasive by endoscopic means, but sometimes surgery is necessary in refractory cases. Techniques employed in the surgical treatment of esophageal strictures include resection of the stenotic esophageal segment or esophageal substitution procedures. Esophageal anastomosis has always been a challenge in pediatric surgery. Anastomosis complications are linked to anatomical, biological and technical aspects. Mechanical tension between esophageal ends is an important cause of complications including anastomotic leaks or dehiscence. Eleven cases of esophageal stenoses, surgically treated in the Pediatric Surgery Department of Emergency Clinical
\end{abstract}

Correspondence to: Dr Radu-Iulian Spătaru, Department of Pediatric Surgery, 'Marie S. Curie' Emergency Clinic Hospital for Children, 20 Constantin Brâncoveanu Boulevard, 041451 Bucharest, Romania

E-mail: radu_spataru@yahoo.com

${ }^{*}$ Contributed equally

Key words: esophageal stenosis, stricture, dilatation, leakage, anastomosis device
Hospital for Children 'Marie S. Curie' by a single team in 5 years, were included in the present study. The results showed that, the main causes of esophageal stenosis were represented by corrosive esophageal injury in five cases, complications of esophageal atresia repair in three cases, congenital esophageal stenosis in two cases and chemotherapy-induced esophageal necrosis in acute lymphoblastic leukemia treatment in one case. The authors also designed and presented a device facilitating esophageal anastomosis under tension. Its principle involved temporary absorption of tension at secure points of the two esophageal pouches and reallocating it in equal amounts following anastomosis while decreasing any stretch-related tissue trauma. In conclusion, this auxiliary tool is beneficial for esophageal anastomosis; however, the standard steps of the esophageal anastomosis procedure should still be considered when necessary.

\section{Introduction}

In contrast to the adult population, the pediatric patient is subject to continuous, dynamic growth and physiological changes. Therefore, treating and thus preventing dysphagia in children should also be considered vital subject matter of pediatrics since aspiration may lead to life-threatening complications and failure to thrive or malnourishment can generate serious repercussions on both the intellectual and physical development of the latter adult (1). Dysphagia appears to be a consequence of an esophageal motility disorder or a 
craniofacial malformation, but often is the result of a narrowing of the esophageal lumen, a condition that may be congenital or acquired. Congenital esophageal stenosis is present from birth but may not be associated with dysphagia in early life. Acquired esophageal strictures primarily occur following esophageal anastomoses in esophageal atresia. Other causes of acquired esophageal obstructions are caustic injuries, peptic or inflammatory disorders (2).

As mentioned in Spitz (3), to emphasize the frailty of an esophageal anastomosis in children, Potts reported in 1950 that, 'to anastomose the ends of an infant's esophagus, the surgeon must be as delicate and precise as a skilled watchmaker; no other operation offers a greater opportunity for pure technical artistry' (3). The anastomosis technique in esophageal atresia (EA) has been a controversial subject since the early years of modern pediatric surgery. In 1968, Cloud (4) was clear on the issue: 'What is the best way to do an anastomosis?' In the same article, Cloud revealed his personal communications with Potts over tips and tricks in esophageal anastomosis, with Potts claiming that using ductus clamps to hold up both esophageal segments tension-free while placing all the sutures before releasing them, constituted a favorable step in order to avoid trauma by excessive stress over the first stiches (4).

Anastomotic leakage is directly related to tension of the suture and albeit minor or consecutive to major dehiscence; it represents one of the most frequent and threatening complications of esophageal atresia repair (5). In the early ages of EA surgery this event had an extremely adverse effect on the survival rate varying from 30 to $60 \%$ (4), data that justified the surgeon's anxiety over esophageal anastomosis tension which is obviously variable and related to the gap length between the upper and lower esophageal pouch. At present, anastomotic leak incidence varies from 4 to $16 \%$ and is associated with significant morbidity and mortality (6).

Considerable breakthroughs were made with regard to EA patient prognosis and survival. The surgical and intensive care management of EA patients allowed centers to report an overall survival rate of $75 \%$ (in the 1980 s) to $100 \%$ (in 2009) in cases with a birth weight over 2,000 g) and without major cardiac malformations (7,8). Alongside this evolution, Puri et al reported in 1981 the opportunity of a delayed primary anastomosis considering a spontaneous postnatal growth of the two esophageal segments (9), concluding in 1992, following the first series of long-term positive outcomes applying this principle that, 'the best esophagus is the patient's own esophagus and, therefore, every effort should be made toward esophageal preservation by delayed primary anastomosis'. Together with Puri's observations (10), the use of esophageal substitution procedures started to cease (11). Instead of this approach, primary esophageal anastomosis became the primary technique utilized although the challenge of the long esophageal gap remained. Consequently, willing to preserve the native esophagus by all means, surgeons opt for anastomotic tension even in long gap EA, which constitutes a risk factor for leakage, dehiscences or esophageal strictures. In addition, anastomotic tension may displace the gastroesophageal junction, leading to incompetence of the gastric cardia and consecutive high gastroesophageal reflux, adding to the risk of recurrent stricture formation or anastomotic leakage (12-14).
Besides tension, other factors have been incriminated to precipitate anastomotic leaks in EA including small, fragile lower segments, esophageal wall ischemia at the two esophageal ends, suture technique and materials used, excessive mobilization of the distal end and increased gap length (14-16). In extremely rare cases, the trachea-esophageal fistula may integrate duplication cysts; thus, ischemia or tissue sacrifice may be inevitable, creating a common esophageal gap into a longer gap, thereby manifesting challenges in the use of the anastomosis technique and decision-making (17). Nevertheless, even if at first glance low birth weight and prematurity are considered risk factors for anastomotic complications, recent findings do not show any correlation between these risk factors $(18,19)$. Regarding anastomotic complications and the surgical approach, postoperative morbidity following thoracoscopy appears to be insufficiently studied and reported. In addition, thoracotomy should remain the gold standard in the absence of experienced teams and careful patient selection (20).

Classically, anastomosis under tension is made by dividing it into a posterior layer and an anterior layer, each of it being closed separately after placing a set of 5/0,6/0 interrupted sutures on each side. Progressive tension is applied on one half until upper and lower esophageal segments stick together and then stitches are tied (21).

\section{Patients and methods}

The aim of this study was is to present our experience in the surgical treatment of esophageal strictures on 11 cases. The ages of the patients ranged from 3 to 12 years with a mean age of 7 years. All the cases included in the study presented symptomatic esophageal strictures in which the minimally-invasive approach failed or it was not indicated and presented to the Pediatric Surgery Department over a period of 5 years. Patient medical charts were retrospectively reviewed for causes of strictures, imagistic tools used, surgical solutions and results. All the patients were treated by a single pediatric surgical team at the 'Marie S. Curie' Emergency Clinical Hospital for Children in Bucharest.

Subsequently, a novel device was designed and registered at the State Office of Inventions and Trademarks (no. 4/87/30.04.2014) recommending it for reducing the tension in the esophageal anastomosis when required. Informed consent was obtained in all cases and the study obtained ethics approval (no. 16460/2021) from the 'M.S. Curie' Children's Hospital Ethical Council.

\section{Results}

General. Ten consecutive cases of esophageal strictures were identified: in 5 cases these were secondary to corrosive esophageal injury, 3 cases were complications of esophageal anastomosis in EA repair, 2 cases were congenital esophageal stenosis and 1 case was the consequence of chemotherapy-induced esophageal necrosis and ulcers in acute lymphoblastic leukemia treatment. In all cases dysphagia was the cardinal symptom.

Esophageal strictures secondary to caustic injury in the 5 cases. The mean age at surgery was 70 months for the 


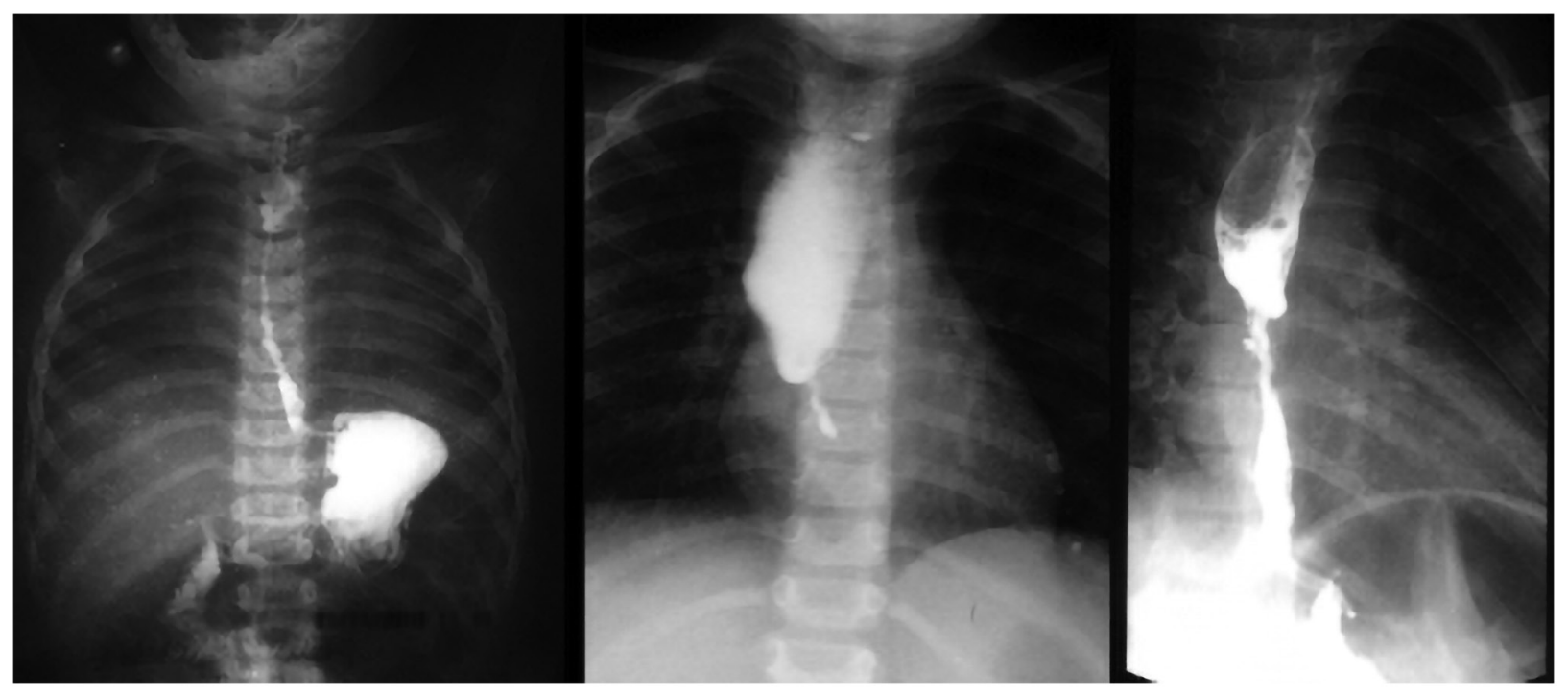

Figure 1. Barium swallow presenting long esophageal strictures secondary to caustic injury.

5 cases. The youngest patient was 3 years of age and the eldest was aged 12 years. All 5 cases underwent Stamm gastrostomy at the acute phase of the corrosive injury and repeated sessions of bougienage dilations were initiated. In one case, esophageal fistula and mediastinitis occurred. After at least 1 year of conservative treatment attempts retrosternal isoperistaltic transverse colon esophageal replacement was the procedure of choice. The main reason for selecting this solution was the long extension (Fig. 1) of the stricture, which was unmanageable by bougienage. Early complications included 1 case of leakage at proximal esophageal-colic anastomosis which was managed conservatively.

Esophageal strictures secondary to esophageal anastomosis in EA repair in 2 cases. Three patients had surgery at the age of 4,5 , and 7 years, all three being cases with a poor follow-up after primary esophageal repair and subsequently failed dilatation procedures. Resection and re-anastomosis of the esophagus was considered. In one case, Nissen fundoplication and gastrostomy was initially performed because of high gastro-esophageal reflux with presenting high risk of chronic aspiration.

Congenital esophageal stenosis in 2 cases. Two cases of congenital esophageal stenoses were included in our series. The first case was a 4-month-old female admitted for repeated episodes of regurgitation, initially considered as gastroesophageal reflux. An esophagogram was performed showing the narrowing of the esophagus at T4 level and aspiration of the barium in the bronchial tree (Fig. 2). Bougienage dilatation was carried out but without any benefits. Resection of the stenotic segment $(\sim 2 \mathrm{~cm})$ and end-to-end anastomosis was performed. Histopathological examination of the resected esophagus revealed fibro-muscular thickening of the esophageal wall.

The second case of congenital esophageal stenosis was a 2-year-old male who was admitted in the Department of Pediatric Surgery for repeated episodes of regurgitation. The esophagogram showed a tight, long, esophageal segment (Fig. 3). The patient also had associated trisomy

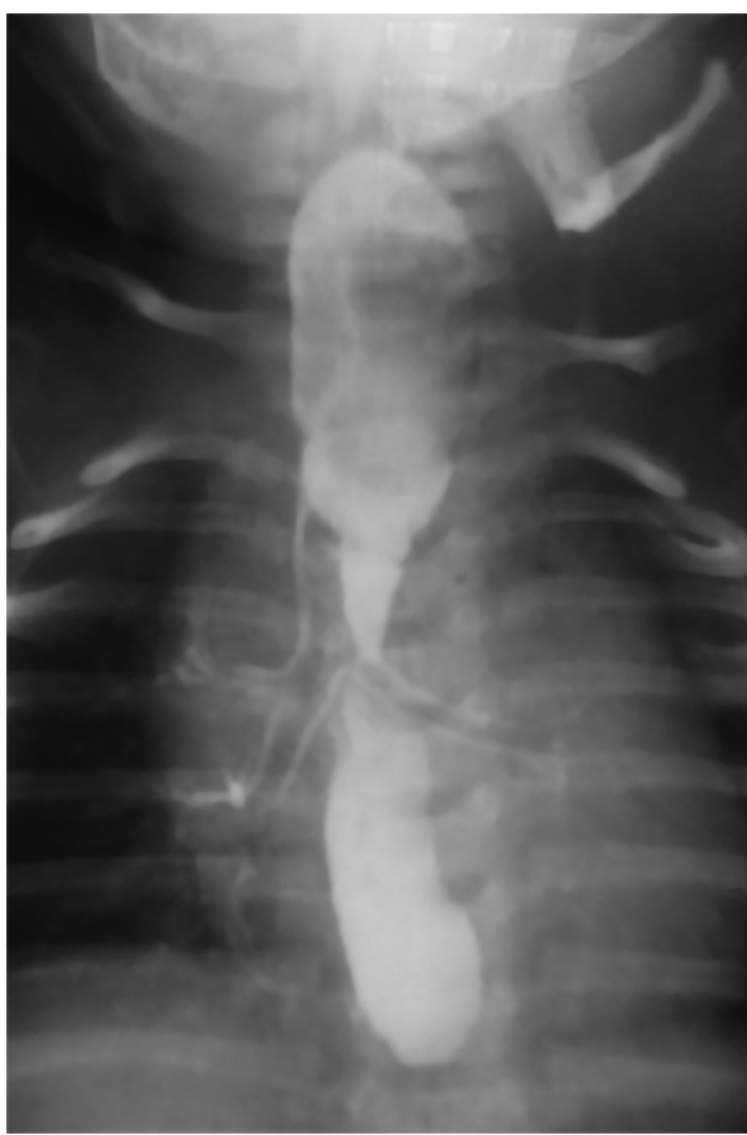

Figure 2. Esophagogram of congenital esophageal stenosis. Tracheal and bronchus invasion of contrast substance are also evident.

21 , and was previously operated for ventricular septal defect and cleft palate. Bougienage was attempted without any results. Lower esophageal substitution with distal ileum was performed with a favorable outcome.

Chemotherapy-induced esophageal ulcer and necrosis complicated with esophageal stricture in 1 case. A 4 year-old-girl 


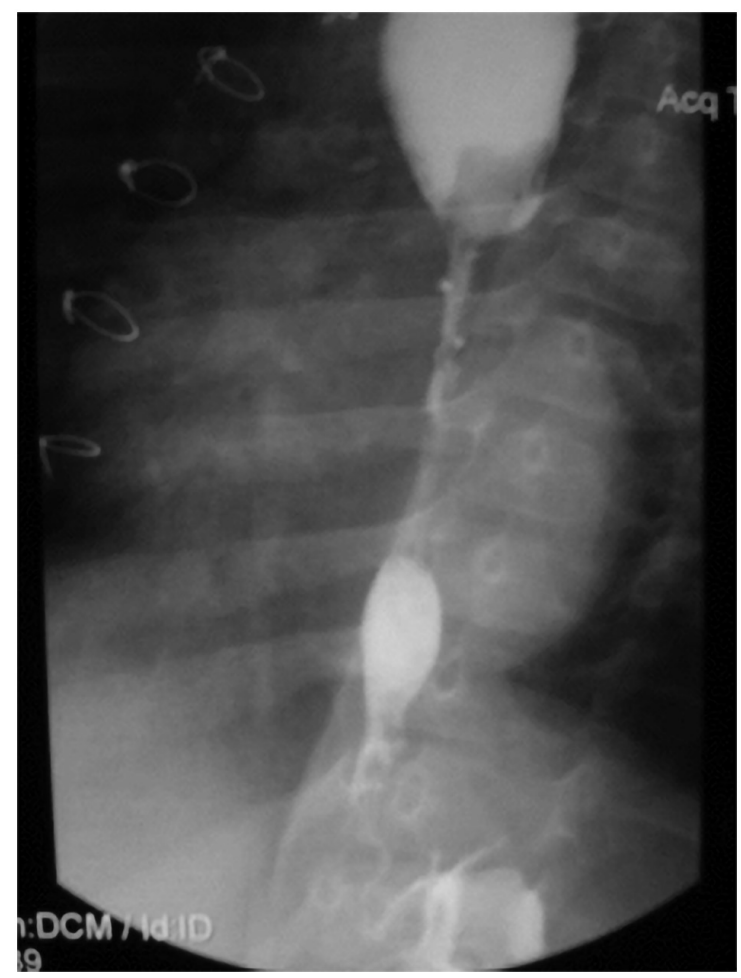

Figure 3. Esophagogram showing congenital esophageal stenosis as a narrowing of distal esophagus. Proximal dilatation of the upper third is evident.

was referred to the Department of Pediatric Surgery by the oncologist for evaluation of achronic dysphagia. She was previously treated, starting at 2 years of age, for acute lymphoblastic leukemia. High doses of dexamethasone were used in the leukemia treatment and she also had a history of systemic candidiasis. We presumed the esophageal narrowing etiology was multifactorial in this situation. Resection of the stenotic segment objectified on the esophagogram and esophageal anastomosis was performed with favorable outcome.

Tension-releasing device for end-to-end esophageal anastomosis. Technically, the device we developed is a metallic structure following the contour of a half-cylinder divided by a longitudinal plane (a ditch-like skeleton). Each of the semi-circular cylinder base halves has 2 set of coils attached for anchoring the stay sutures (Fig. 4).

The device overtakes the mechanical tension in the two esophageal ends, adjusting it in order to place all the sutures tension-free (or under minimal tension) and releasing the tension only after the complete anastomosis is achieved; therefore, the force is divided and equally allocated on each of the sutures.

After dissection of the two esophageal ends is completed, the device is inserted into the thoracic cavity, parallel to the esophagus and over the anastomosis site. The surgeon divides the anastomosis into two quadrants by placing two stay sutures $(\mathrm{S})$ on each esophageal ending, through the adventitia and muscularis of the esophageal wall $(\mathrm{K})$, respecting the submucosal layer, at a relative distance from the two esophageal endings that will further constitute the final anastomosis. The stay sutures are stretched and fixed to the coils of the device (C), each pair in opposite directions thereby narrowing the gap

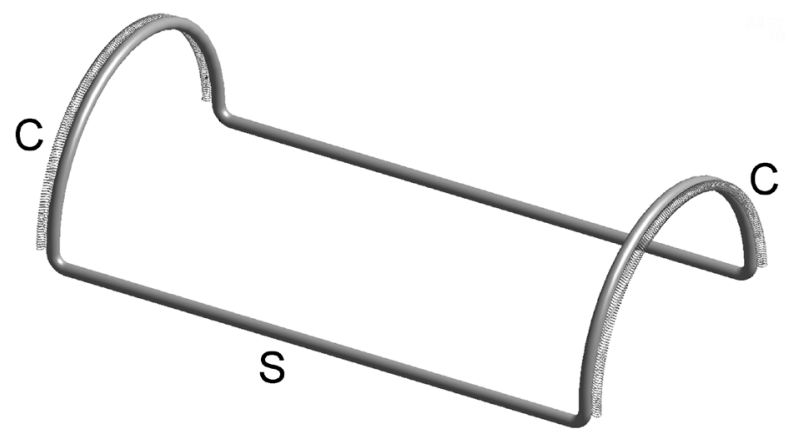

Figure 4. Tension-releasing device for end-to-end esophageal anastomosis: metallic structure $(\mathrm{S})$ with attached coils for suture anchoring $(\mathrm{C})$.

between the two esophageal endings without applying any kind of mechanical effort on the future site of the anastomosis (Fig. 5). The full anastomosis is then performed without any tension, the stay sutures are removed individually and the device is removed allowing each of the anastomosis sutures to overtake a small amount of tension equal to the others.

We conceived this considering that unequally divided tension, ischemia and esophageal mucosal or submucosal tears during surgical manipulation are significant factors in anastomotic leaks and dehiscence incidence. Equidistant and uniform forces in the anastomoses may prevent stricture formation or may attribute benign aspects to the esophageal stricture.

\section{Discussion}

Esophageal stenosis in children is congenital or acquired. Congenital esophageal stenosis may embrace various histopathological aspects. These types of stenosis can be secondary to tracheo-bronchial remnants, fibro-muscular hypertrophy of the esophagus or they can present as a membranous diaphragm in the wall of the esophagus. Minimally invasive treatment of congenital esophageal stenosis, depending on their nature, varies widely, including dilatations of the esophagus, endoscopic resection of the diaphragm or longitudinal myotomy. Invasive surgery should be considered in cases where endoscopy is ineffective (22).

Secondary esophageal stenoses in pediatric population are mainly encountered as a complication of esophageal anastomoses in EA. Other causes of acquired esophageal obstructions are caustic injuries, peptic or inflammatory disorders (2). Chemotherapy in acute leukemia of childhood is known to induce esophageal ulcer or necrosis. Moreover, during administration of oncologic treatments, the altered immune status led to esophageal candidiasis. Regarding the side effects of the drugs administered, esophageal strictures in acute leukemia are related to esophagitis as a consequence of high-dose dexamethasone, doxorubicin, citarabin and methotrexate (23).

Regardless of the etiology of esophageal stenosis, when surgical treatment is required to preserve the native esophagus and to reduce tension between the two esophageal ends making anastomosis possible, use of several techniques and procedures has been suggested to narrow the gap length. Some of them can be applied while attempting both initial and 
Table I. Techniques of esophageal reconstruction.

Proximal esophagomyotomy (circular or spiral): using or without using balloon catheters to facilitate mobilization Upper pouch (and lower pouch) bougienage

Using magnetic attraction between the two pouc

Bridging the gap with sutures between the two esophageal ends and progressively reducing the gap

Extrathoracic elongation of the upper pouch by progressively migrating a proximal esophagostomy down the chest wall

Placing traction sutures on both the proximal and distal esophageal pouches, externally or internally

Creating a full-thickness anterior flap from the upper pouch which can be tubularized and anastomosed to a lower esophageal segment

Gastric transposition into the chest; resection of the distal esophagus, division of the left gastric vessels and moving the fornix upwards through the diaphragmatic hiatus, where it will be anastomosed to the proximal esophagus; pyloroplasty systematically performed.

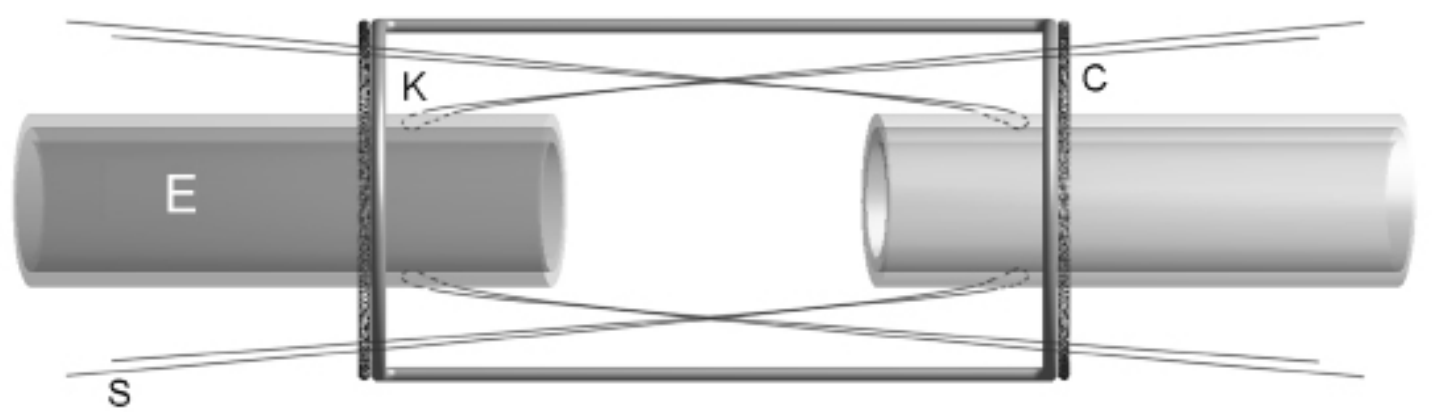

Figure 5. Anchoring (K) the esophageal ends (E) and fixing them to the device's coils (C) using stay sutures (S), thus allowing esophageal anastomosis with minimal tension.

delayed anastomosis, while others are reserved for delayed primary esophageal repair (Table I). All these are reported more or less with associated morbidities, prolonged hospital stays, or requirement of multiple surgical reinterventions (24). Such interventions are possible in tertiary centers, having well-trained multidisciplinary teams for congenital and acquired various difficult/rare conditions $(25,26)$.

In addition to previously mentioned preoperative or intraoperative surgical techniques whose purpose is gap narrowing or releasing the anastomosis tensions, other postoperative approaches are used, including keeping the neck flexed which reduces esophageal stretching or keeping up to one-week mechanical ventilation together with elective paralysis (27). Other methods have been reported to be useful in an isolated case series, including using a trans-anastomotic balloon catheter passed into the stomach and then, subsequent to a gentle force from outside the gastroesophageal junction, reducing the anastomotic tension, the tube is fixed externally to the skin (28).

Besides endoscopic dilatations, using balloon or bougienage may be considered, esophageal self-expandable stents placing may be considered in case of recurrent strictures; however, most patients may experience chest pain, nausea or stent migration. Esophageal stenting in pediatric patients is reported to have successful results or is considered to be a bridge to definitive surgery $(2,29)$.

For all our patients, we obtained an appropriate informed consent from their parents $(30,31)$.

The tension-releasing device for end-to-end esophageal anastomosis was designed due to its advantages including that, it builds up a high quality anastomosis permitting an improved overview on the symmetry of the surgical suturing, eliminates from the operatory field (albeit extremely small) the use of unnecessary forceps employed in suture tying leading to an improved view and more space for maneuvers, and allows optimal and synchronous stretching on the common longitudinal axis of both esophageal endings. However, there are disadvantages to consider including, the suture passing through auxiliary parts of the esophageal wall, unnecessary mainly in anastomosis, thus the possibility of unwanted laceration (although this may depend solely on the surgeon's skill), the device should fit to each patient's size, and the present tool cannot be used by surgeons who prefer the thoracoscopic approach.

In conclusion, esophageal stenosis of childhood may present by various anatomic aspects depending on their underlying etiology. Case selection should always be carefully made. Treatment should initially follow a conservative endoscopic approach. If refractory, surgery should be considered and depending on characteristics of strictures it can consist of esophagomyotomy, stenotic area resection followed by end-to-end anastomosis or esophageal replacement procedures. The surgical procedure should always be adapted on a careful evaluation of the stricture appearance and the patient's history. When the gap between the esophageal ends resulting from removal of the lesion is long, esophageal anastomosis is challenging. The tool we designed may be useful in this situation, reducing the mechanical tension when performing the anastomosis. 


\section{Acknowledgements}

Not applicable.

\section{Funding}

No funding was received.

\section{Availability of data and materials}

The datasets used and/or analyzed during the current study are available from the corresponding author on reasonable request.

\section{Authors' contributions}

DAI and RIS performed surgical procedures and confirm the authenticity of all the raw data. OS, LFT and AT conducted the preoperative investigations; IB, RIS and DR contributed to the literature review; NB, IB and OS analyzed the data; RIS, AT, FG and FF contributed to critical revisions on the intellectual content; DAI finally revised the draft of the manuscript. All authors read and approved the final version of the manuscript.

\section{Ethics approval and consent to participate}

Informed consent from the parents/legal guardians was obtained in all cases and the study obtained ethics approval from the 'M.S. Curie' Children's Hospital Ethical Council, registered with no. 16460/2021.

\section{Patient consent for publication}

Parents/legal guardian consent for publication of images or clinical data was obtained.

\section{Competing interests}

The authors declare that they have no competing interests.

\section{References}

1. Dodrill P and Gosa MM: Pediatric dysphagis: Physiology, assessment, and management. Ann Nutr Metab 66 (Suppl 5): S24-S31, 2015.

2. Vandenplas Y: Management of benign esophageal strictures in children. Pediatr Gastroenterol Hepatol Nutr 20: 211-215, 2017.

3. Spitz L: Oesophagealatresia. Orphanet J Rare Dis 2: 24, 2007.

4. Cloud DT: Anastomotic technic in esophageal atresia. J Ped Surg 3: 561-564, 1968.

5. D'Urzo C, Bunuomo V, Rando G and Pintus C: Major anastomotic dehiscence after repair of esophageal atresia: Conservative management or reoperation? Dis Esophagus 18: 120-123, 2005.

6. Gupta M, Mahajan JK, Bawa M and Rao KLN: Esophageal atresia and tracheoesophageal fistula: Effect of pleural cover on anastomotic dehiscence. J Indian Assoc Pediatr Surg 16: 50-53, 2011.

7. Spitz L, Kiely EM, Morecroft JA and Drake DP: Oesophageal atresia: At-risk groups for the 1990s. J Ped Surg 29: 723-725, 1994.

8. Okamoto T, Takamizawa S, Arai H, Bitoh Y, Nakao M, Yokoi A and Nishijima E: Esophageal atresia: Prognostic classification revisited. Surgery 145: 675-681, 2009.

9. Puri P, O'Donnell B and Guiney EJ: Delayed primary anastomosis following spontaneous growth of esophageal segments in esophageal atresia. J Ped Surg 16: 180-183, 1981.
10. Puri P, Ninan GK, Blake NS, Fitzgerald RJ, Guiney EJ and O'Donnell B: Delayed primary anastomosis for esophageal atresia: 18 Months' to 11 years' follow-up. J Pediatr Surg 27: 1127-1130, 1992

11. Kelly JP, Shackelford GD and Roper CL: Esophageal replacement with colon in children: Functional results and long-term growth. Ann Thorac Surg 36: 634-643, 1983.

12. Sri Paran T, Decaluwe D, Corbally M and Puri M: Long-term results of delayed primary anastomosis for pure oesophageal atresia: A 27-year follow up. Pediatr Surg Int 23: 647-651, 2007.

13. Nagaya M, Kato J, Niimi N, Tanaka S and Iio K: Proposal of a novel method to evaluate anastomotic tension in esophageal atresia with a distal tracheoesophageal fistula. Pediatr Surg Int 21: 780-785, 2005.

14. Mckinnon LJ and Koloske AM: Prediction and prevention of anastomotic complications of esophageal atresia and tracheoesophageal fistula. J Pediatr Surg 25: 778-781, 1990.

15. Upadhyaya VD, Gangopadhyaya AN, Gupta DK, Sharma SP, Kumar V, Pandey A and Updhyaya AD: Prognosis of congenital tracheoesophageal fistula with esophageal atresia on the basis of gap length. Pediatr Surg Int 23: 767-771, 2007.

16. Chittmittrapap S, Spitz L, Kiely EM and Brereton RJ: Anastomotic leakage following surgery for esophageal atresia. J Pediatr Surg 27: 29-32, 1992.

17. Spataru RI, Popoiu MC and Ivanov M: Foregut duplication cys associated with esophageal atresia-one-stage neonatal surgical repair. Indian J Surg 77 (Suppl 1): S52-S55, 2015.

18. Teimourian A, Donoso F, Stenström P, Arnadottir H, Arnbjörnsson E, Lilja $\mathrm{H}$ and Salö M: Gender and birth weight as risk factors for anastomotic stricture after esophageal atresia repair: A systematic review and meta-analysis. BMC Pediatr 20: 400, 2020.

19. Dingemann C, Brendel J, Wenskus J, Pirr S, Schukfeh N and Reinshagen K: Low gestational age is associated with less anastomotic complications after open primary repair of esophageal atresia with tracheoesophageal fistula. BMC Pediatr 20: 267, 2020.

20. Laberge JM and Blair GK: Thoracotomy for repair of esophageal atresia: Not as bad as they want you to think! Dis Esophagus 26: 365-371, 2013.

21. Spitz L and Pierro A: Operative Pediatric Surgery. Spitz L and Coran A (eds). CRC Press, Inc., Boca Raton, FL, pp135, 2013.

22. Takamizawa S, Tsugawa C, Naruaki M, Satoh S, Kanegawa K, Nishijuma E and Muraji T: Congenital esophageal stenosis: Therapeutic strategy based on etiology. J Pediatr Surg 37: 197-201, 2002.

23. Kelly K, Storey L, O'Sullivan M, Butler K, McDermott M, Corbally M, McMahon C, Smith OP and O'Marcaigh A: Esophageal strictures during treatment for acute lymphoblastic leukemia. J Pediatr Hematol Oncol 32: 124-127, 2010.

24. Harmon CM and Coran AG: Congenital anomalies of the esophagus. In: Pediatric Surgery. Coran AG, Adzick NS, Krummel TM, Laberge J, Shamberger RC and Caldmone AA (eds). 7th edition. Elsevier Saunders, Philadelphia, PH, pp893-918, 2012.

25. Suciu N, Serban A, Toader O, Oprescu D and Spataru RI: Case report of fetal lingual tumor-perinatal care and neonatal surgical intervention. J Matern Fetal Neonatal Med 27: 314-319, 2014.

26. Spătaru RI, Iozsa DA and Ivanov M: Preputial calculus in a neurologically-impaired child. Indian Pediatr 52: 149-150, 2015.

27. Pinheiro PF, Simões e Silva AC and Pereira RM: Current knowledge on esophageal atresia. World J Gastroenterol 18: 362-372, 2012.

28. Boia ES, Nicodin A, Popoiu MC, Trailescu M and David VL: An effective method to release anastomotic tension after repair of esophageal atresia using a Foley catheter. Chirurgia (Bucur) 108: 189-192, 2013

29. Tandon S, Burnand KM, De Coppi P, McLaren CA, Roebuck DJ and Curry JI: Self-expanding esophageal stents for the management of benign refractory esophageal strictures in children: A systematic review and review of outcomes at a single center. J Pediatr Surg 54: 2479-2486, 2019.

30. Serban D, Spătaru RI, Vancea G, Bălăsescu SA, Socea B, Tudor C and Dascălu AM: Informed consent in all surgical specialties: From legal obligation to patient satisfaction. Rom J Leg Med 28: 317-321, 2020.

31. Serban D, Smarandache AM, Cristian D, Tudor C, Duta L and Dascălu AM: Medical errors and patient safety culture-shifting the healthcare paradigm in Romanian hospitals. Rom J Leg Med 28: 195-201, 2020 . 